\title{
Kunitomo Sakurai, Solar Neutrino Problems - How they were Solved
}

Hans J. Haubold ${ }^{1,2^{*}}$ and Arak M. Mathai ${ }^{1,3}$

${ }^{1}$ Centre for Mathematical and Statistical Sciences, Peechi Campus, KFRI, Peechi-680653, Kerala, India

${ }^{2}$ Office for Outer Space Affairs, United Nations, P.O. Box 500, Vienna International Center, A-1400 Vienna, Austria

${ }^{3}$ Department of Mathematics and Statistics, McGill University, 805 Sherbrooke Street West, Montreal, Quebec, Canada

"Corresponding author: Hans J. Haubold, Office for Outer Space Affairs, United Nations, P.O. Box 500, Vienna International Center, A-1400 Vienna, Austria, Tel: +43-1-260 60 4950; E-mail: hans.haubold@gmail.com

Rec date: May 28, 2015; Acc date: June 01, 2015; Pub date: June 05, 2015

Copyright: @ 2015 Haubold HJ, et al. This is an open-access article distributed under the terms of the Creative Commons Attribution License, which permits unrestricted use, distribution, and reproduction in any medium, provided the original author and source are credited.

\section{Book Review}

The central message of Sakurai's outstanding book is: The Sun is a surface source of photons. Not in perfect hydrostatic equilibrium the Sun oscillates with periods to be understood similar to seismic waves. As a volume source of neutrinos, created in the proton-proton chain and the CNO cycle reactions, spectroscopy allows a direct look into the current reaction rates operating in the solar core. Solar surface and volume phenomena show a wide range of variations and modulations that are not yet well understood and may lead to the discovery of new fundamental solar, nuclear, and/or elementary particle physics. A particular case is the omnipresent quasi-biannual variation discovered by Sakurai. Sakurai intentionally leaves open the question concerning the possible origin of the Sun's neutrino heartbeat illustrated at https:// www.youtube.com/watch?v=qgg4YUnLL3Y. So-called standard models of the Sun and of elementary particles may experience significant modification as one gradually understands better the solar surface and volume phenomena through future observations of the Sun (Figure 1).

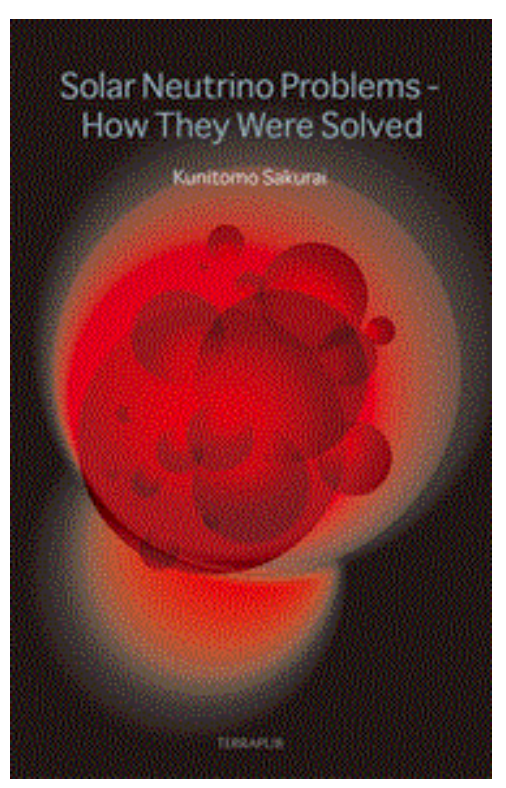

Figure 1: Physics: Gravitationally Stabilized Solar Fusion Reactor

Sakurai impressively recalls the current understanding of the Sun as a star in terms of basic physics. The internal structure of the Sun is determined by conditions associated with mass conservation, momentum conservation, energy conservation, and specific modes of energy transport throughout the Sun. Much of what has been observed and theorized about the Sun can be accounted for in terms of basic physical laws: Newton's laws of gravity and motion, the first two laws of thermodynamics, Einstein's law of the equivalence of mass and energy, Boyle's law, Charles' law of perfect gases, and Heisenberg's uncertainty principle. The theory of the structure and evolution of the Sun has been formulated in the first half of the twentieth century; it is connected with names like Lane, Emden, Schwarzschild, Eddington, Chandrasekhar, Hoyle, and Fowler. In the second half of the twentieth century, theories about the Sun were greatly refined; this is due in part to new observation techniques and to computer simulations of its structure and evolution. The overall picture of the internal structure of the Sun seems to be well understood and is shown in the following Figure 2.

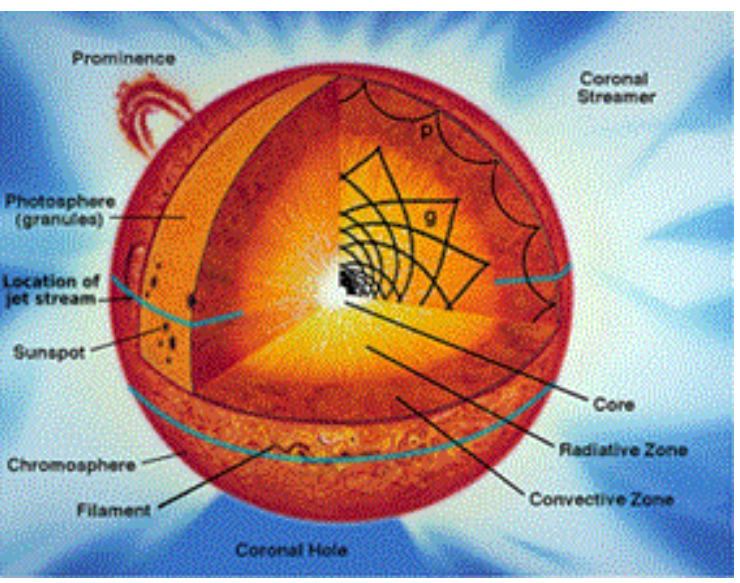

Figure 2: Seismology: Solar waves

When considering its internal structure, rotation and magnetic fields can be neglected in a first approximation since the Sun is spherically symmetric. Oscillations in the Sun are damped so that they die out within a few periods. However, interference between these localized waves produces global standing waves, also known as normal modes. Solar oscillation modes are essentially divided up into three categories, depending on the restoring force that drives them: acoustic, gravity, and surface-gravity wave modes.

The p-mode or acoustic waves have pressure as their restoring force. Their dynamics are determined by the variation of the speed of sound inside the Sun. The g-mode or gravity waves are density waves which have gravity (negative buoyancy of displaced material) as their restoring force. The g-mode oscillations are confined to the interior of the Sun below the convection zone and are practically unobservable at 
the surface. The restoring force is caused by adiabatic expansion: in the deep interior of the Sun, the temperature gradient is weak and a small packet of gas that moves upward will be cooler and denser than the surrounding gas, and will therefore be pulled back to its original position; this restoring force drives the g-modes. In the solar convection zone, the temperature gradient is slightly greater than the adiabatic lapse rate, so that there is an anti-restoring force (that drives convection) and g-modes cannot propagate. The g-modes are evanescent through the entire convection zone, and are thought to have residual amplitudes of only millimeters at the photosphere, though more prominent as temperature perturbations. The f-mode or surface gravity waves are occurring at or near the photosphere, where the temperature gradient again drops below the adiabatic lapse rate. Sakurai shows that many solar surface and volume phenomena due to the above waves are linked to the quasi-biannual periodicity, even the solar neutrino flux measured in solar neutrino observatories (Figure 3).

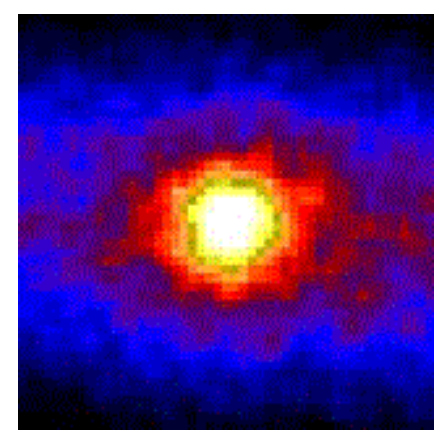

Figure 3: Spectroscopy: Solar neutrinos.

For several decades there was a large discrepancy between the theory of how the Sun shines and the most direct experimental test of this theory. This discrepancy was called 'the solar neutrino problem'; it refers to the fact that the Sun is a volume source of neutrinos which are particles produced by thermonuclear reactions in the deep interior of the Sun, and that the copious flux of solar neutrinos predicted by theory did not match the flux detected by observatories on Earth (the above figure shows the neutrino image of the Sun). This problem has been studied experimentally by R. Davis Jr. and theoretically by J.N. Bahcall since the 1960s. It was understood that solar neutrinos are the only particles that have the ability to travel from the core of the Sun to its surface almost without interaction with solar matter, escaping freely into space carrying the most direct information about physical processes in the deep solar interior. Neutrinos were first proposed by W. Pauli in 1930, but their existence wasn't solidly confirmed experimentally until 1956 by C.L. Cowan and F. Reines. Neutrinos interact weakly with other particles, so it took a nuclear reactor to produce enough neutrinos to be detected in the laboratory. Neutrino observatories (Homestake, SAGE, GALLEX+GNO, SuperKamiokande, SNO, Borexino), sensitive enough to observe solar and astrophysical neutrinos, are large arrays of detectors. The original solar neutrino problem meant either the understanding of solar and nuclear physics were significantly off, or the understanding of particle physics was wrong. Since the understanding of particle physics was based upon laboratory experiments, it was thought that the standard solar model must be flawed. There were several attempts to reformulate solar fusion models to account for the smaller neutrino rate, going so far as to propose that fusion in the Sun's core had shut down temporarily. But over time these alternatives became increasingly untenable. Observational data supported the traditional solar fusion model and therefore the neutrino physics must be incorrect. The physics of elementary particles is described by what is also known as a particle physics' standard model. This model unified the understanding of electromagnetism and the nuclear forces known as the strong and weak interactions. According to this standard model, the three types of neutrinos (electron, muon, tauon) should be massless. In 1968 B. Pontecorvo proposed that neutrinos had a small mass. This proposal would change the standard model slightly, but leave the overall predictions largely unchanged. The mass of the neutrinos couldn't be large, otherwise they would have already been observed. But even the tiniest amount of mass would mean neutrinos could not move at the speed of light, and could change flavors. This flavor-changing effect is known as neutrino oscillations. Neutrino oscillations would explain the solar neutrino problem as experimentally confirmed by SuperKamiokande and SNO in 2001 and 2002. The neutrinos produced in the Sun's core would all be electron neutrinos, but as they moved through the Sun to its surface they would oscillate between the various types. By the time they reached the Earth, the neutrinos would be an equal mix of each type, meaning that only a third would be electron neutrinos. Since only the electron neutrinos were measured, the neutrino number would be a third that predicted by the standard model and detected by Home stake, SAGE, and GALLEX+GNO.

However, the phenomenon of solar neutrino oscillations is complicated by the interaction of neutrinos with matter as they are streaming through the Sun. The three flavors interact differently with matter and Mikheyev, Smirnov, and Wolfenstein proposed an effect, today named MSW effect, where neutrino oscillations are differently enhanced depending on their energies. This MSW effect is negligible at low energies (sub-MeV), where vacuum oscillations dominate, while at high energies in the $\mathrm{MeV}$ range the production of neutrinos is suppressed considerably. A transition is predicted between these two energy ranges where a clear difference between vacuum oscillation and matter oscillation should be observed in the solar neutrino observatories operating today (SuperKamiokande, SNO, Borexino). Remarkably, current data do not confirm clearly the expected signature of the MSW effect and leave room for a number of different models to explain nonstandard neutrino interaction and propagation. Additionally, Sakurai emphasizes the fact that the data from those solar neutrino observatories show variation and modulation when exposed to various methods of harmonic analysis. Future research will have to face this fact. 